\title{
Optimizing timing performance of CdTe detectors for PET
}

This content has been downloaded from IOPscience. Please scroll down to see the full text.

Download details:

IP Address: 131.227.23.35

This content was downloaded on 06/09/2017 at 10:03

Manuscript version: Accepted Manuscript

Nakhostin

To cite this article before publication: Nakhostin, 2017, Phys. Med. Biol., at press: https://doi.org/10.1088/1361-6560/aa8664

This Accepted Manuscript is: (c) 2017 Institute of Physics and Engineering in Medicine

During the embargo period (the 12 month period from the publication of the Version of Record of this article), the Accepted Manuscript is fully protected by copyright and cannot be reused or reposted elsewhere.

As the Version of Record of this article is going to be / has been published on a subscription basis, this Accepted Manuscript is available for reuse under a CC BY-NC-ND 3.0 licence after the 12 month embargo period.

After the embargo period, everyone is permitted to copy and redistribute this article for non-commercial purposes only, provided that they adhere to all the terms of the licence https://creativecommons.org/licences/by-nc-nd/3.0

Although reasonable endeavours have been taken to obtain all necessary permissions from third parties to include their copyrighted content within this article, their full citation and copyright line may not be present in this Accepted Manuscript version. Before using any content from this article, please refer to the Version of Record on IOPscience once published for full citation and copyright details, as permission will likely be required. All third party content is fully copyright protected, unless specifically stated otherwise in the figure caption in the Version of Record.

When available, you can view the Version of Record for this article at: http://iopscience.iop.org/article/10.1088/1361-6560/aa8664 


\title{
Optimizing timing performance of CdTe detectors for PET
}

\author{
M. Nakhostin \\ Department of Physics, University of Surrey, Guildford GU2 7XH, UK \\ E-mail: M.nakhostin@surrey.ac.uk \\ Tel.:+44 1483686113
}

Fax: +44 1483686781

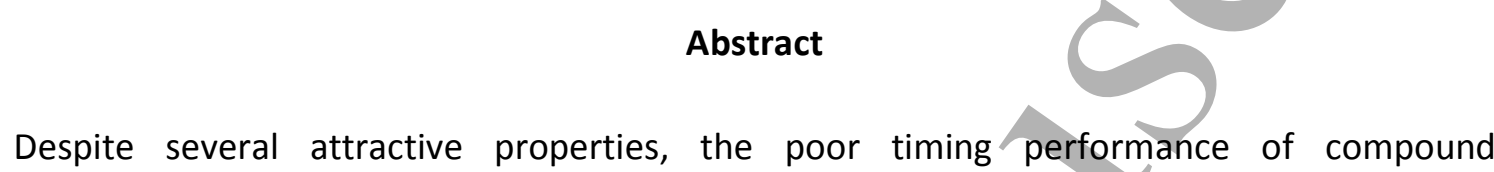
semiconductor detectors such as CdTe and CdZnTe has hindered their use in commercial PET imaging systems. The standard method of pulse timing with such detectors is to employ a constant-fraction discriminator (CFD) at the output of a timing filter which is fed by the pulses from a charge-sensitive preamplifier. The method has led to a time resolution of about $10 \mathrm{~ns}$ at full-width at half-maximum (FWHM) with $1 \mathrm{~mm}$ thick CdTe detectors. This paper presents a detailed investigation on the parameters limiting the timing performance of Ohmic contact planar CdTe detectors with the standard pulse timing method. The jitter and time-walk errors are studied through simulation and experimental measurements and it is revealed that the best timing results obtained with the standard timing method suffer from a significant loss of coincidence events ( $50 \%)$. In order to improve the performance of the detectors with full detection efficiency, a new digital pulse timing method based on a simple pattern recognition technique was developed. A time resolution of $3.29 \pm 0.10 \mathrm{~ns}$ (FWHM) in the energy range of 300-650 keV was achieved with an Ohmic contact planar CdTe detector $\left(5 \times 5 \times 1 \mathrm{~mm}^{3}\right)$. The digital pulse processing method was also used to correct for the chargetrapping effect and an improvement in the energy resolution from $4.83 \pm 0.66 \%$ to $2.780 \pm 0.002 \%$ (FWHM) at $511 \mathrm{keV}$ was achieved. Further improvement of time resolution through a moderate cooling of the detector and the application of the method to other detector structures are also discussed.

Keywords: Cadmium Telluride; Semiconductor detectors; PET; Time resolution; Energy resolution 


\section{Introduction}

Compound semiconductor detectors such as cadmium telluride (CdTe) and cadmium zinc telluride (CZT) have attracted a considerable interest for use in high-resolution positron emission tomography (PET) systems (Ishii et al 2007, Mitchell et al 2008, Peng and Levin 2010a, Morimoto et al 2011). The attraction of these detectors is mainly due to their inherent high spatial resolution, good energy resolution, direct detection of $\gamma$-rays and the possibility of achieving a high packing fraction of the detectors (>99 \%) (Vaska et al 2005, Zhang et al 2005, Kikuchi et al 2005, Peng and Levin 2010b, Gu et al 2011, Groll et al 2017). So far, by using stacks of thin CdTe detectors ( $1 \mathrm{~mm}$ thick), a few prototype PET systems have been built demonstrating $\sim 1 \mathrm{~mm}$ spatial resolution (Ishii et al 2007, Ueno et al 2009, Morimoto et al 2011). However, even with thin detectors, the timing performance of the detectors is poor as compared to that of the conventional scintillator-based PET detectors. A good time resolution is required to reject random events thus reducing noise in the/reconstructed PET images. The reported time resolutions at room temperature are about 10 ns (FWHM) (Baldazzi et al 1993, Giboni et al 2000, Okada et al 2002, Vaska et al 2005, Nakhostin et al 2009) which is 2-3 times larger than that for slow scintillator detectors such as NaI(TI) and BGO. Moreover, the spectroscopic performance of compound semiconductor detectors is still limited by the charge-trapping effect (Eisen et al 1999). Studies have suggested that, in principle, the timing and spectroscopic performances of the detectors can be improved by using the information in the shape of output pulses (Meng and He 2005, Mitchell et al 2008, Nakhostin et al 2010). However, the common front-end electronic systems based on analog application specific integrated circuits (ASICS) offer very limited flexibility to exploit the information in the shape of pulses. On the other hand, digital front-end systems based on free-running analog-to-digital converters offer enormous flexibility in the pulse processing of radiation detectors. In digital systems, since the shape of each pulse is known, a proper pulse processing algorithm can be used to improve the performance of the detectors by exploiting the pulse-shape information. The previous attempts to improve the timing performance of semiconductor detectors by using digital techniques have been based on fitting the measured waveforms (Meng and $\mathrm{He}$ 2005, Mitchell et al 2008, Groll et al 2017), using rise-time information (Nakhostin et al 2010) and comparing the detector pulse shape with a set of reference pulses (Crespi et al 2010). In this paper, we first perform a simulation study on the parameters affecting the timing performance of a CdTe detector with the standard pulse timing method of semiconductor $\gamma^{-}$ 
ray detectors that includes the application of a CFD to the outputs of a timing filter amplifier which is fed by the pulses from a charge-sensitive preamplifier. The simulation study is followed by an experimental analysis of the timing performance of a planar Ohmic contact $\mathrm{CdTe}$ detector by using digital pulse processing techniques. Particular attention is paid to the effect of the pulse timing procedure on the efficiency of coincidence measurements which is critical for PET application. Finally, a new digital timing method based a simple pattern recognition technique is proposed to significantly improve the timing performance of the CdTe detector while maintaining the full detection efficiency. The pattern recognition technique is also used to improve the spectroscopic performance of the detector.

\section{Simulation results}

The shape of the induced charge pulse on the electrodes of a planar CdTe detector can be expressed by using the Hecht's relation (Hecht, 1932) as:

$$
Q(t)=\frac{e N_{\circ} V}{w^{2}}\left\{\mu_{e} \tau_{e}\left[1-\exp \left(\frac{-t}{\tau_{e}}\right)\right]+\mu_{h} \tau_{h}\left[1-\exp \left(\frac{-t}{\tau_{h}}\right)\right]\right\}
$$

where $e$ is the charge of an electron, $N_{\circ}$ is the number of initial charge carriers, $w$ is the width of the detector, $V$ is the bias voltage, $\mu$ is the mobility, $\tau$ is the carrier lifetime and the $e$ and $h$ subscripts represent electrons and holes, respectively. Charge carriers will only contribute to the induced charge during their drift times:

$$
t_{e}=\frac{\left(w-x_{\mathrm{o}}\right) w}{\mu_{e} V} \text { and } t_{h}=\frac{x_{\mathrm{o}} w}{\mu_{h} V}
$$

where $x_{\circ}$ is the interaction location measured from the cathode. Due to the significant difference in the mobility of electrons and holes, different interaction locations of $y$-rays in the detector lead to variable contributions from electrons and holes, thereby producing variable pulse-shapes. The shape of charge pulses can be further influenced by the rise-time of the charge-sensitive preamplifier whose transfer function can be expressed as:

$$
\frac{V(s)}{Q(s)}=-A \frac{s \tau_{d}}{s \tau_{d}+1} \frac{1}{s \tau_{r}+1}
$$

where $A$ is the gain of the system, $\tau_{r}$ is the rise-time of the preamplifier, $\tau_{d}$ is the decay-time of the preamplifier and $s$ is the Laplace variable. We used the z-transform of the transfer function to calculate the preamplifier output pulses in the discrete time domain. 

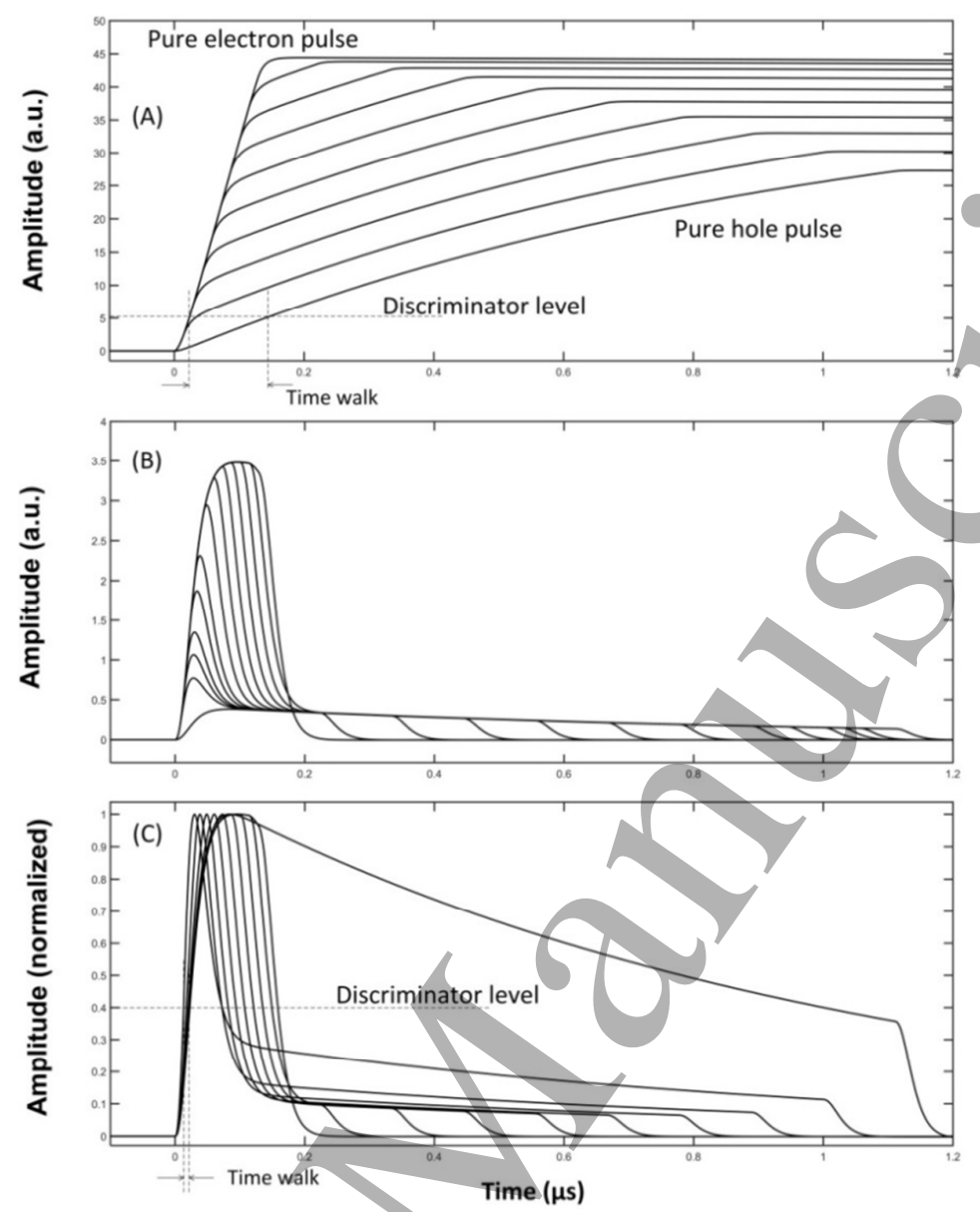

Figure 1. (A) Simulated preamplifier output pulses. A trigger on the electron component of the pulses minimizes the time jitter due to the larger slope of the electron component of the pulses and avoids the large time-walk error due to the change in the slope of the pulses. (B) Pulses after a timing filter with 10 ns integration time constant and 50 ns differentiation time constant. In spite of the constant slope of the electron component of the original preamplifier pulses, the slope and amplitude of the filtered pulses varies from event to event, depending on the amount of electron component. (C) Normalized filtered pulses and a typical constant-fraction discriminator equal to $40 \%$ of the pulses' amplitudes. A time-walk error due to the variable rise-time of the pulses results. The error can be reduced by lowering the discriminator level.

Figure 1(A) shows noiseless preamplifier output pulses calculated for interactions at different locations in a 1-mm thick CdTe detector operating at 100 volt of bias voltage. The rise-time and decay-time of the preamplifier were, respectively, $5 \mathrm{~ns}$ and $80 \mu \mathrm{s}$ and the charge transport parameters were $\mu_{e}=950\left(\mathrm{~cm}^{2} \mathrm{~V}^{-1} \mathrm{~s}^{-1}\right), \mu_{h}=73\left(\mathrm{~cm}^{2} \mathrm{~V}^{-1} \mathrm{~s}^{-1}\right), \tau_{e}=1.2 \times 10^{-6}$ (s) and $\tau_{h}=4.6 \times 10^{-6}(\mathrm{~s})$, according to the data published for CdTe crystals grown by Acrorad Ltd., Japan (Iniewski 2010). When actual preamplifier pulses are fed into a time discriminator circuit, there are two main sources of timing errors in marking the arrival-time of pulses: 
jitter and walk. Jitter is the time uncertainty that is caused by the electronic noise and statistical fluctuations of the detector's pulses in crossing the threshold level of the timing discriminator. If $\sigma_{n}$ is the root-mean square ( $\mathrm{rms}$ ) value of the electronic noise superimposed on the pulse and $d V / d t$ is the slope of the pulse when its leading-edge crosses the discriminator threshold level, the timing resolution due to the electronic noise is given by the noise-to-slope ratio as (Wilmshurst 1985):

$$
\sigma_{t}=\frac{\sigma_{n}}{(d V / d t)}
$$

As seen in figure $1(A)$, the slope of the electron-component of the pulses is much higher than that for the hole-component of the pulses, leading to smaller time jitter. The time-walk error is the time movement of the output time pick-off relative to the input pulse due to the variations in the shape and amplitude of the input pulses. From Figure $1(A)$ one can see that a large time-walk happens when the trigger level lies on the hole-component of the pulses, and thus, minimization of both jitter and time-walk errors dictates to set the trigger on the electron component of the pulses. Although due to the finite range of primary electrons in the detector practically all pulses contain some contribution from electrons (Nakhostin and Esmaili-Torshabi 2015), pulses resulting from interactions close to the anode may contain a small electron component that means the discriminator level should be set as low as possible. However, the lower level of a discriminator is practically limited by the level of electronic noise. Therefore, the use of a timing filter is necessary to reduce the noise level while maintaining the pulses' slope. In the discrete time domain, a timing filter can be implemented by using digital differentiator and integrator algorithms. If one assumes the preamplifier pulses are sampled with a sampling interval of $\Delta T$, from the z-transfer function of an $R C$ integrator, the following recursive formula can be obtained for a digital integrator:

$$
y[n]=\alpha \cdot x[n]+(1-\alpha) \cdot x[n-1],
$$

where $x[n]$ is the sampled preamplifier pulse, $y[n]$ is the integrated pulse, $n$ is the sample index and $\alpha$ is a constant given by $\Delta T /(\Delta T+\tau)$ in which $\tau$ is the integration time constant of the integrator. Similarly, a recursive formula for a digital differentiator was obtained as:

$$
z[n]=\beta \cdot z[n-1]+\beta \cdot(y[n]-y[n-1]),
$$

where $z[n]$ is the differentiated pulse, $y[n]$ is the input pulse and $\beta$ is a constant given by $\tau_{1} /\left(\Delta T+\tau_{1}\right)$ in which $\tau_{1}$ is the differentiation time constant. The effect of timing filter with 
typical integration time constant of $10 \mathrm{~ns}$ and differentiation time constant of $50 \mathrm{~ns}$ is shown in Figure $1(B)$. The timing filter shortens the duration of pulses according to the differentiation time constant of the filter but if the duration of the fast component of the pulse is shorter than the differentiation time constant, the leading-edge of the pulse ends before the time constant of the differentiator and the hole-component appears in the trailing-edge of the pulse. For pulses with large electron-component, the leading-edge of the filtered pulse continues until the differentiator cuts the pulse. Therefore, pulses with a small electron-component show a shorter rise-time than pulses with larger electron-component. On the contrary, the amplitude of the pulses reduces with decreasing the contribution of electrons that consequently reduces the signal-to-noise ratio of the filtered pulses. The variations in the rise-time and amplitude of the filtered pulses result in time-walk error. By using a standard CFD, the error due to the variations in the amplitude of the pulses is effectively removed but the error due to the variations in the rise-time of the pulses remains. This is shown in Figure $1(\mathrm{C})$ where the filtered pulses are normalized to mimic the behaviour of a constant-fraction discriminator. It is apparent that, in spite of the constant slope of the electron-component of the original preamplifier pulses, the slope of the filtered pulses varies from event to event, depending on the amount of electron component. This produces a time-walk error which can be reduced by lowering the discrimination threshold but this is ultimately limited by the signal-to-noise ratio of the pulses, i.e. the amount of electron component. In standard analog pulse timing with semiconductor detectors, the time-walk is minimized by setting the shaping delay on the analog CFD less than the minimum rise-time of the filtered pulses to make the zero-crossing time of the CFD pulse early in the pulse lifetime. This mode of CFD operation is called the amplitude and rise-time compensated (ARC) mode and was originally used with germanium detectors (Chase 1968, Cho and Chase 1972).

\section{Experimental results}

\subsection{Experimental setup}

The experimental setup consists of a planar CdTe detector $\left(5 \times 5 \times 1 \mathrm{~mm}^{3}\right)$ placed against a $\mathrm{LaBr}_{3}(\mathrm{Ce})$ scintillator detector. The CdTe detector has Ohmic contacts (platinum electrodes), fabricated by Acrorad Ltd., in Japan. The detector was used with a fast charge-sensitive preamplifier (model A250 from Amptek Inc). The preamplifier has a rise-time of $2.5 \mathrm{~ns}$ at 
zero input capacitance. The CdTe detector was operated at room temperature with a bias voltage of 100 volt. Under these operating conditions, the leakage current of the detector was $80 \mathrm{nA}$ and the capacitance of the detector was $2 \mathrm{pF}$. The $\operatorname{LaBr}_{3}(\mathrm{Ce})$ detector has a cylindrical shape of 1" diameter by 1" height, coupled to a Hamamatsu photomultiplier tube of type $\mathrm{H} 10570$. The time resolution of the $\operatorname{LaBr}_{3}(\mathrm{Ce})$ was separately measured to be 380 ps (FWHM) for $511 \mathrm{keV}$-rays. The details on the procedure of time pick-off from the $\mathrm{LaBr}_{3}(\mathrm{Ce})$ detector's signals can be found in a previous study (Nakhostin et al 2014). The coincidence measurements were taken with a ${ }^{22} \mathrm{Na}$ point source $(1.6 \mathrm{MBq})$ placed close to the CdTe detector and at a distance of about $5 \mathrm{~cm}$ from the $\mathrm{LaBr}_{3}(\mathrm{Ce})$ detector. The outputs of the preamplifier and the photomultiplier tube were simultaneously digitized by means of a fast waveform digitizer with 10-bit resolution at $1 \mathrm{GS} / \mathrm{s}$ sampling rate (model DC252HF from Agilent Technologies Inc). The digitizer was triggered by signals from the $\operatorname{LaBr}_{3}(\mathrm{Ce})$ detector and thus all coincidence events were recorded regardless of the shape and energy of pulses from the CdTe detector. In order to maximize the signal-to-quantization noise ratio, the preamplifier's output before feeding into the digitizer was amplified with a fast no-shaping amplifier so that the outputs for $511 \mathrm{keV}$-rays covered almost $80 \%$ of the one volt input range of the digitizer. Four sets of coincidence signal waveforms, each containing approximately 2500 coincidence events, were acquired and transferred to a personal computer for offline analysis with a program written in MATLAB programming environment.

\subsection{Digital pulse processing methods}

The digital time pick-off was carried out in a similar manner to the method described in the simulation approach by using a digital version of the timing filter (Eq. 5 and Eq. 6) and a digital CFD. The digital CFD algorithm searches for the maximum digitized value of a filtered pulse and sets a threshold based on a predefined fraction of the maximum value. The pulse is then analysed to determine when it initially crosses the threshold and the crossing time is taken as the arrival-time of the pulse. The arrival-time of the pulse is linearly interpolated if it falls between the signal samples (Nakhostin et al 2008). In regard to the $\operatorname{LaBr}_{3}(\mathrm{Ce})$ detector, the pulse arrival-time is marked directly with the digital CFD. The pulse processing steps for the extraction of energy information from sampled preamplifier pulses included a digital baseline correction followed by a digital semi-Gaussian pulse shaper (Nakhostin 2011) to minimize the effect of the electronic noise. 


\subsection{Energy scale calibration}

Figure 2 shows the pulse-height spectra of 662 and $511 \mathrm{keV} \mathrm{y}$-rays acquired by the digital processing of the CdTe detector's pulses. The shaping time constant of the pulse shaper is $2.5 \mu \mathrm{s}$. It is apparent that the full-energy peaks have asymmetric shapes with a tail toward the low energy range which is due to the charge-trapping effect. Several analytical functions have been proposed to account for the peak shape observed with CdTe detectors (Gunnink and Arlt 2001, Redus et al 2009). The model proposed by Gunnink and Arlt contains a central Gaussian component, an exponential term for the peak tail and a constant that accounts for background counts. The analytical expression is given by:

$$
f(E)=a_{\circ}+a_{1} \exp \left[a_{2}\left(E-a_{3}\right)\right]+a_{4} \exp \left[-\left(\frac{E-a_{3}}{\sqrt{2} a_{5}}\right)^{2}\right]
$$

where $E$ and $a_{n}$ denote the energy of interaction and fitted parameters, respectively. The relative energy resolution can be then derived as the ratio of $\mathrm{FWHM}=2.35 a_{5}$ to the peak centre, $a_{3}$. The least-squares fit of Eq. 7 to the $511 \mathrm{keV}$ peak yields $a_{3}$ and $a_{5}$ as $a_{3}=511.97 \pm 3.00$ and $a_{5}=14.89 \pm 2.04$ from which a relative energy resolution of $4.83 \pm 0.66 \%$ (FWHM) was calculated. The uncertainty in the relative energy resolution was calculated by adding the fractional uncertainties in $a_{3}$ and $a_{5}$, as obtained from the least-squares fit, in quadrature. The same procedure was applied to $662 \mathrm{keV}$ peak and an energy resolution of $3.39 \pm 0.20 \%$ was obtained. By using the 511 and $662 \mathrm{keV}$ energy peaks, the energy scale of the system was calibrated and an energy window from 300-550 keV was set for the timing measurements.

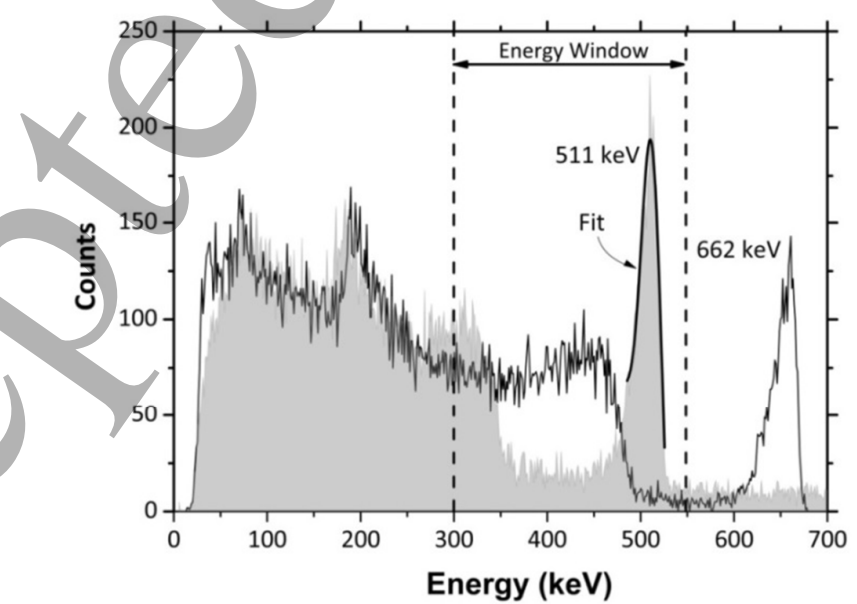

Figure 2. The pulse-height spectra of 662 and $511 \mathrm{keV}$-rays, measured with the CdTe detector biased at 100 volt. The fit of Eq. 7 to the $511 \mathrm{keV}$ peak is also shown. The $R$-square of the fit is $R^{2}=0.93$. The energy resolution of the detector at $511 \mathrm{keV}$ is $4.83 \pm 0.66 \%$ (FWHM). 


\subsection{Timing performance}

Figure 3 shows sample pulses representing $y$-ray interactions at different locations in the detector. The charge transport times are reflected in the leading-edge of the pulses. By using the data published for the charge transport parameters of CdTe crystals fabricated by Acrorad Ltd (Iniewski 2010), the electron and hole transit times at room temperature are calculated to be, respectively, $0.105 \mu$ s and $1.370 \mu$ s at a bias voltage of 100 volt.

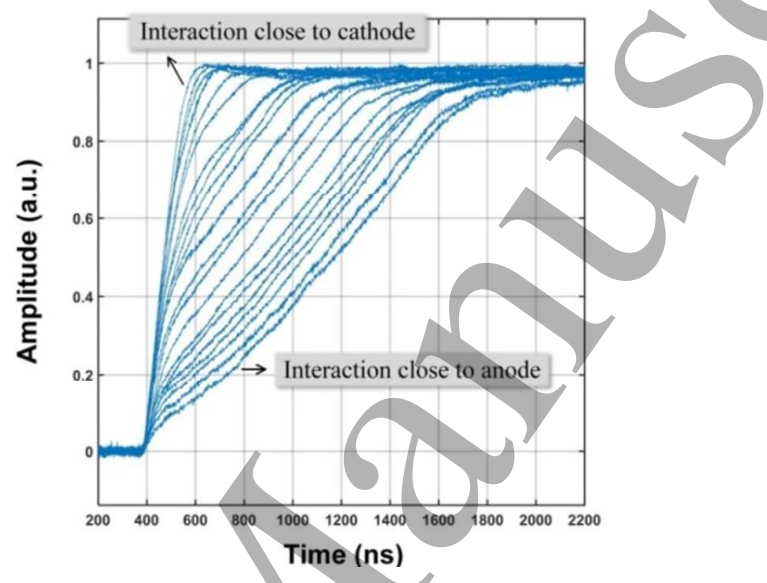

Figure 3. Sample preamplifier pulses from the CdTe detector. The interactions at the surface of the cathode and anode represent, respectively, pure electron and hole signals.

The time spectrum was obtained as histogram of the calculated difference in the arrival-times of pulses from the $\mathrm{CdTe}$ and $\mathrm{LaBr}_{3}(\mathrm{Ce})$ detectors. Due to the superior time resolution of the $\mathrm{LaBr}_{3}(\mathrm{Ce})$ detector, the FWHM of the time spectrum is considered to be entirely due to the time resolution of the CdTe detector. To mark the arrival-time of pulses from the CdTe detector, we set the CFD threshold level at $10 \%$ of the amplitude of each filtered pulse and, by using a trial-and-error procedure, the integration time constant of the timing filter was optimized for various differentiation time constants. It was found that a small integration time constant of $5 \mathrm{~ns}$ gives the best result for all the differentiation time constants but the time resolution strongly varies with the differentiation time constant. Figure 4 shows the time spectra obtained by using $10 \mathrm{~ns}$ and $250 \mathrm{~ns}$ differentiation time constants. The time spectra have an asymmetric shape that can be well fitted with an exponentially modified Gaussian function (Origin 2010). The FWHMs of the fitted functions indicate that by decreasing the differentiation time constant from 250 ns to $10 \mathrm{~ns}$, the time resolution improves from $8.22 \pm 0.12 \mathrm{~ns}(\mathrm{FWHM})$ to $4.01 \pm 0.32 \mathrm{~ns}(\mathrm{FWHM})$. However, it is immediately clear that this is 
achieved at the expense of a significant loss in the number of recorded coincidence events ( $\sim 50 \%)$. Figure 5 shows the effect of the differentiation time constant on the number of recorded coincidence events. The number of events was calculated as the number of events lying in a coincidence time window of 30 ns around the centre of each peak. The number of recorded coincidence events increases by increasing the time constant of the differentiator and approaches its maximum value as the differentiation time constant is increased beyond after 250 ns. At smaller differentiation time constants, a significant number of coincidence events are lost so that with a differentiation time constant of 10 ns the coincidence measurement efficiency is less than $50 \%$. The loss in the number of coincidence events is explained by the fact that for a pulse with small electron component, a short differentiation time constant results in a pulse with poor signal-to-noise ratio. The event then may be lost by a false triggering of the CFD on the baseline noise as shown in figure 6 . The effect of false triggering can be reduced by increasing the CFD fraction but the time resolution significantly degrades due to the increase in the time-walk error and also larger noise-to-slope ratio. The improvement in the time resolution at small differentiation time constants is also due to the rejection of slow pulses that has obviously serious consequences in PET applications and may explain why the reported sensitivities of the prototype PET systems are less than the theoretically expected values (Ishii et al 2007, Morimoto et al 2011). 


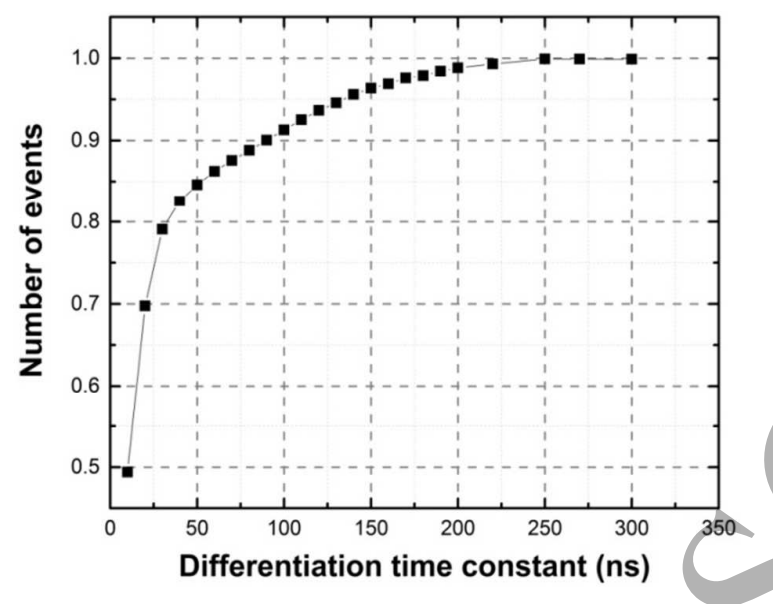

Figure 5. The effect of the differentiation time constant on the number of recorded coincidence events. About $50 \%$ of the coincidence events are lost when the differentiation time constant is only $10 \mathrm{~ns}$.

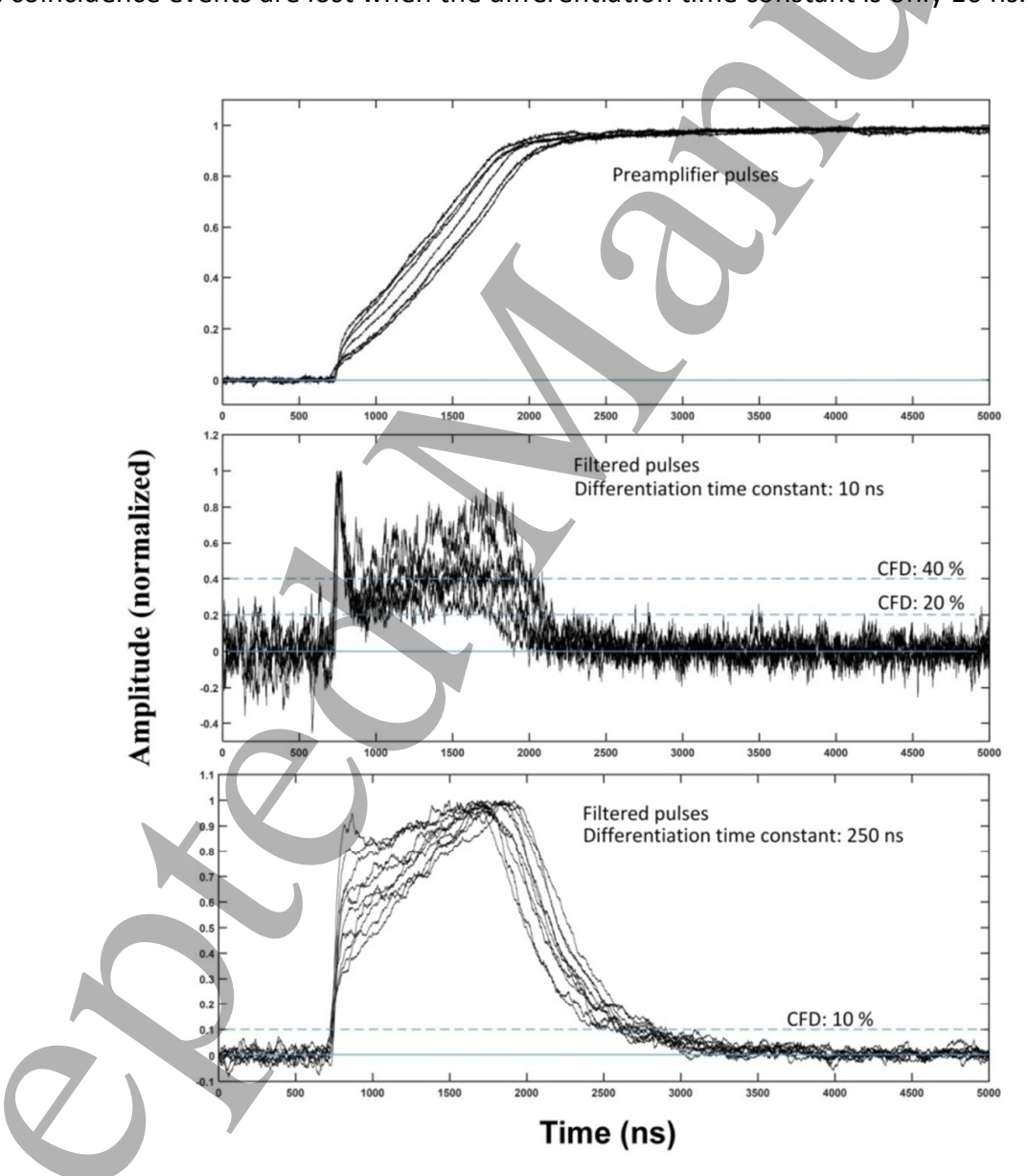

Figure 6. The effect of differentiation time constant and CFD fraction on the efficiency of coincidence measurements. (A) The raw preamplifier pulses with a small electron-component, (B) The normalized pulses after a differentiator with $10 \mathrm{~ns}$ time constant. A CFD fraction of below $40 \%$ lies in the noise level and some events are lost. (C) The normalized pulses after a differentiator with $250 \mathrm{~ns}$ time constant. With $250 \mathrm{~ns}$ of differentiation time constant, the signal-to-noise ratio significantly improves and a CFD fraction of $10 \%$ of pulse amplitude is well above the noise level. 
Another important parameter in the time response of CdTe detectors is the shape of the time spectrum. One can see in figure 4 that the time spectrum has an asymmetric shape with a tail in its right side. The asymmetric shape of the time spectrum results from time-walk of the CFD output as it was discussed in the simulation results. The tail of the spectrum can be quantified by its full-width at tens-maximum (FWTM). The FWTM of the time spectrum obtained with $250 \mathrm{~ns}$ differentiation time constant is about $15 \mathrm{~ns}$ that leads to the loss of coincidence events when a time window based on the FWHM of the spectrum is set. In addition to jitter and walk, timing errors may also result from the imperfections in the electronics system such as inadequate resolution and sampling rate of the digitizer. However, these effects are assumed to be negligible in the current study based on the results of our measurements with fast scintillator detectors (Nakhostin et al 2014).

\section{Performance improvement of the detector}

\subsection{The pattern recognition method}

It was shown in the previous sections that the time response of CdTe detectors is affected by the time-walk error resulting from variable rise-times of the filtered pulses. Since the risetime of a filtered pulse depends on the contribution of electrons in the induced charge pulse, the information in the time profile of the preamplifier pulses can be used to quantify the amount of time-walk. In the past, pulse-shape discrimination methods such as rise-time measurement and double pulse shaping technique have been used to discriminate between pulses of different rise-time (Richter and Siffert 1992, Seino et al 2011). Here, we have used a simple similarity measure to accurately quantify the pulse-shape information. Similarity measures are powerful pattern recognition techniques for quantifying the similarity between data sets presented by vectors. Many similarity measures have been proposed for different applications among which the cosine similarity measure is a popular choice due to its simple implementation (Cha 2007). This method has been already implemented in analog domain by using several peak-sensing ADCs (Takenaka et al 1998). The cosine similarity measure calculates the angle between two vectors $X$ and $Y$ as:

$$
\operatorname{Cos}(\theta)=\frac{X \cdot Y}{|X||Y|}=\frac{\sum_{i=1}^{p} x_{i} y_{i}}{\sqrt{\sum_{i=1}^{p} x_{i}^{2}} \times \sqrt{\sum_{i=1}^{p} y_{i}^{2}}}
$$


where $X \cdot Y$ is a scalar product of the vectors, $|X|$ and $|Y|$ are, respectively, the norm of the vectors, $x_{i}$ and $y_{i}$ are the elements of the vectors, $p$ is the number of vector's elements and $\theta$ is the angle between the two vectors. The parameter $\cos (\theta)$ reflects how similar are the shape of the two vectors with $\cos (\theta)=1$ for identical vectors. Our approach for quantifying the variations in the shape of pulses from CdTe detectors is based on the calculation of the similarity between sampled preamplifier pulses and an ideal reference pulse, $R(t)$ shown in figure 7. The reference pulse is synthetized according to a $y$-ray interaction at the surface of the cathode under no charge-trapping effects as:

$$
R(t)=\left\{\begin{array}{cc}
\frac{\mu_{e} V}{w^{2}} t & 0 \leq t \leq \frac{w^{2}}{\mu_{e} V} \\
1 & t>\frac{w^{2}}{\mu_{e} V}
\end{array}\right.
$$

where $\mu_{e}$ is the mobility of electrons, $V$ is the bias voltage (100 volt), $w$ is the detector's thickness $(1 \mathrm{~mm})$ and $t$ is the time. The mobility of electrons was assumed to be $950 \mathrm{~cm}^{2} \mathrm{~V}^{-1} \mathrm{~s}^{-1}$ according to the data reported for the same CdTe crystals (Iniewski 2010). For a better illustration of the difference in the shape of the pulses, the pulses shown in figure 7 are normalized to their amplitudes but in the calculations no normalization is required. The similarity measure is calculated by using the samples of $R(t)$ and the pulse under analysis that lie in a time interval starting from a constant-fraction of pulses' amplitudes and ending after a fixed time interval equal to the maximum charge collection time (1370 ns). Figure 8 shows the simulation results for the variations of the time pick-offs against the similarity measure. The simulated preamplifier pulses were generated for a uniform distribution of interaction locations along the detector's $1 \mathrm{~mm}$ gap and the time pick-offs were calculated according to the pulse timing model described in the previous section (CFD fraction $10 \%$, integration time constant $5 \mathrm{~ns}$ and differentiation time constant $250 \mathrm{~ns})$. These results show that the relation between the CFD output and the similarity measure is not linear. It is also apparent that in spite of a uniform distribution of the interaction points along the detector's gap, the number of events in the lower range of the similarity measure is less than the number of events in the upper range which means the similarity measure does not linearly increase with the amount of electron-component. Moreover, in spite of the difference in the shape of the pulses, the same similarity measure is obtained for the events whose hole-component only lies in the time interval used for the similarity measure calculation. The number of such events can be reduced by lowering the CFD fraction that determines the start of the time interval though this 
is practically limited by the level of electronic noise. In our measurements, a CFD fraction equal to $15 \%$ of the amplitude of the pulses was used as it is well above the noise level for pulses in the energy range of 300-550 keV.

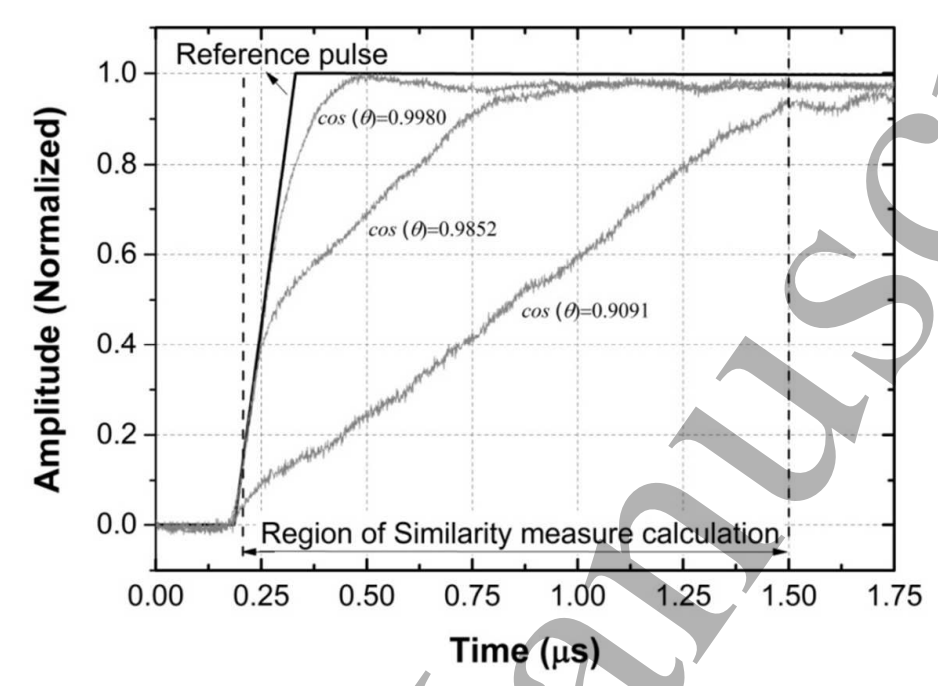

Figure 7. The reference pulse (shown in black) together with some examples of the detector's pulses. The similarity measure is calculated by using the pulses' samples in a time window of $1370 \mathrm{~ns}$, starting from $15 \%$ of the pulses' amplitudes. 


\subsection{Time resolution improvement}

\subsubsection{Time-walk correction}

Figure $9(A)$ shows the scatter plot of the measured time difference between the two detectors against the similarity measure of the CdTe detector's pulses. In the measurements, the differentiation time constant of the timing filter was 250 ns and the CFD threshold was $10 \%$ of a filtered pulse's amplitude to guarantee a detection efficiency close to $100 \%$. One can see that a strong correlation exists between the similarity measure and the time difference between the coincidence pulses. Polynomials of different orders were examined to describe the correlation and it was found that a third order polynomial function well describes the data as assessed by the $R$-square value of the fit $\left(R^{2}=0.92\right)$. The fit function was obtained as:

$$
T(S)=1821.3619 S^{3}-2792.05608 S^{2}+373.18983 S+652.29079
$$

where $T$ is the time difference and $S$ is the similarity measure. It is also observed that some events at the lower range of the similarity measure do not follow the overall trend. These are events whose holes-component only lies in the time interval used for the similarity measure calculation (see Figure 8). Eq. 10 was used to correct for the time-walk error of the CFD output as $\mathrm{CFD}_{\mathrm{c}}=\mathrm{CFD}+(T(1)-T(S))$, where $\mathrm{CFD}_{c}$ is the corrected time pick-off and CFD is the initial time pick-off for the CdTe detector's pulses. The corrected scatter plot is shown in figure 9 (B). The corrected scatter plot was also fitted with a linear function. The fit function, $T(S)=-$ $2.492 S+57.47$, indicates that the time-walk over the whole range of similarity measure is reduced to less than 250 ps. Figure 10 shows the corrected and the original time spectra. The corrected spectrum is well fitted with a Gaussian function $\left(R^{2}=0.98\right)$. The FWHM of the time spectrum improves from $8.22 \pm 0.12$ ns to $3.82 \pm 0.02$ ns and the FWTM improves from 15 ns to 7 ns.

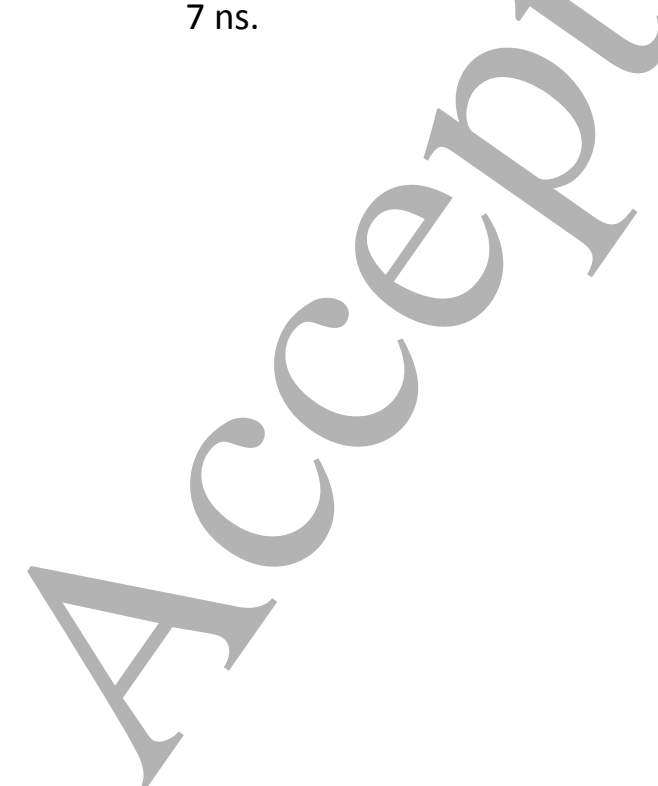




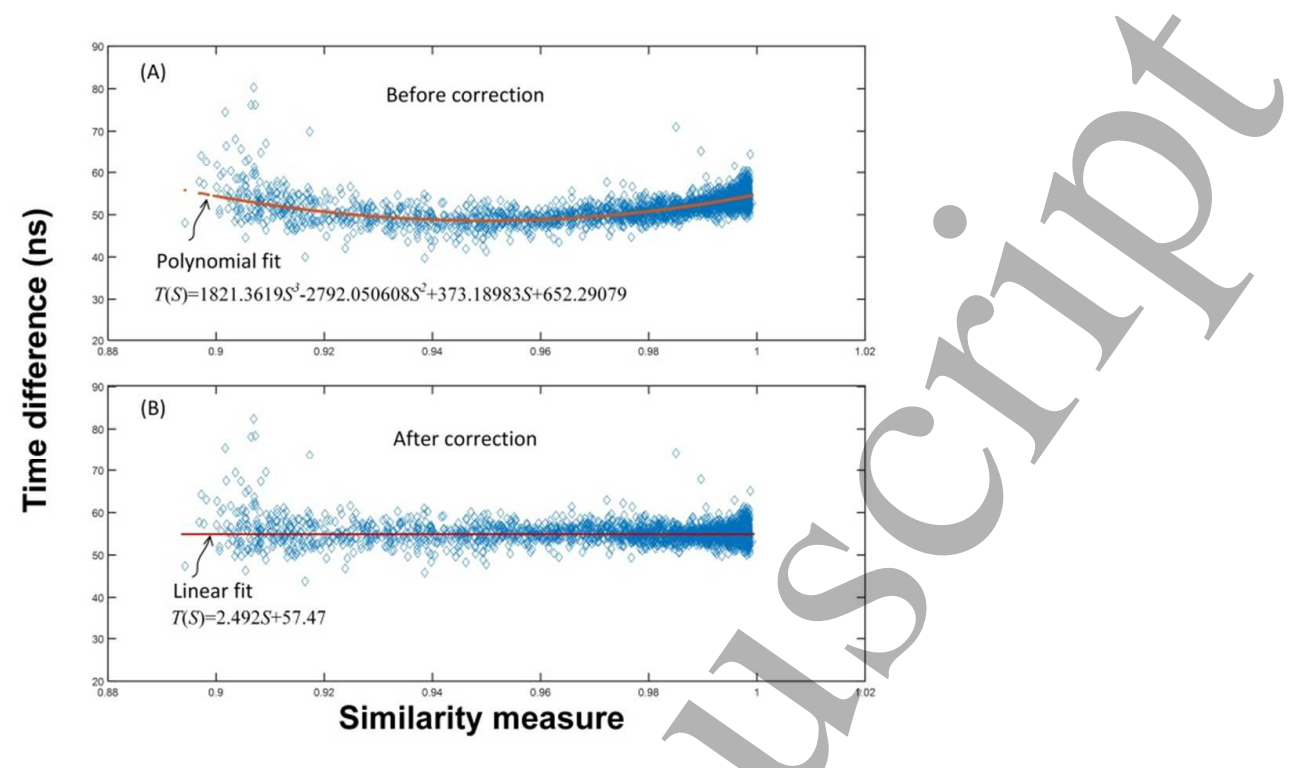

Figure 9. (A) The scatter plot of the measured time difference between the two detectors against the similarity measure of the CdTe pulses. The strong correlation between the time pick-off of CdTe pulses and the similarity measure is apparent and is well described with a third order polynomial $\left(R^{2}=0.92\right)$. (B) The corrected scatter plot. A linear fit to the corrected data yields a function with a very small slope, indicating the insignificance of the time-walk error.

\subsubsection{Time-walk correction with minimization of time jitter}

The simulation results showed that filtered pulses exhibit a variable slope, depending on the contribution of electrons to the pulses, and thus, in addition to time-walk, the amount of 
time jitter will also vary from event to event for a fixed CFD fraction. This means that the jitter can be reduced by applying a CFD fraction according to the shape of the pulse as reflected in the similarity measure. To do so, the pulses were divided into five groups of similarity measures and the CFD threshold was optimized for each group. The timing filter parameters were $5 \mathrm{~ns}$ of integration and $10 \mathrm{~ns}$ of differentiation. The choice of small differentiation time constant is because the effect of leakage current noise reduces at smaller differentiation time constants. The effect of the small differentiation time constant is then compensated by applying a sufficiently high CFD threshold to avoid false triggering on the baseline noise. The results of CFD optimization for each group of pulses are shown in figure 11. The optimum CFD fraction for slow pulses $(0.9 \leq S \leq 0.92)$ is $60 \%$ compared to $18 \%$ for fast pulses $(0.98 \leq S \leq 1)$. The best time resolution is achieved for the fast pulses as $2.69 \pm 0.04 \mathrm{~ns}$ (FWHM). To obtain the overall time spectrum, the centroids of all time spectra, determined by fitting Gaussian functions, were aligned by adding a correction factor to the CFD outputs of each group of pulses. The resulting time spectrum is shown in figure 12 . The time spectrum has a time resolution of $3.29 \pm 0.10$ ns (FWHM) which shows more than sixty percent improvement over the original time spectrum $(8.22 \pm 0.12 \mathrm{~ns})$. This timing performance is in the same range or superior to that of slow scintillators such as sodium iodide (Nal(TI)) and bismuth germinate (BGO) (Eriksson et al 2004).
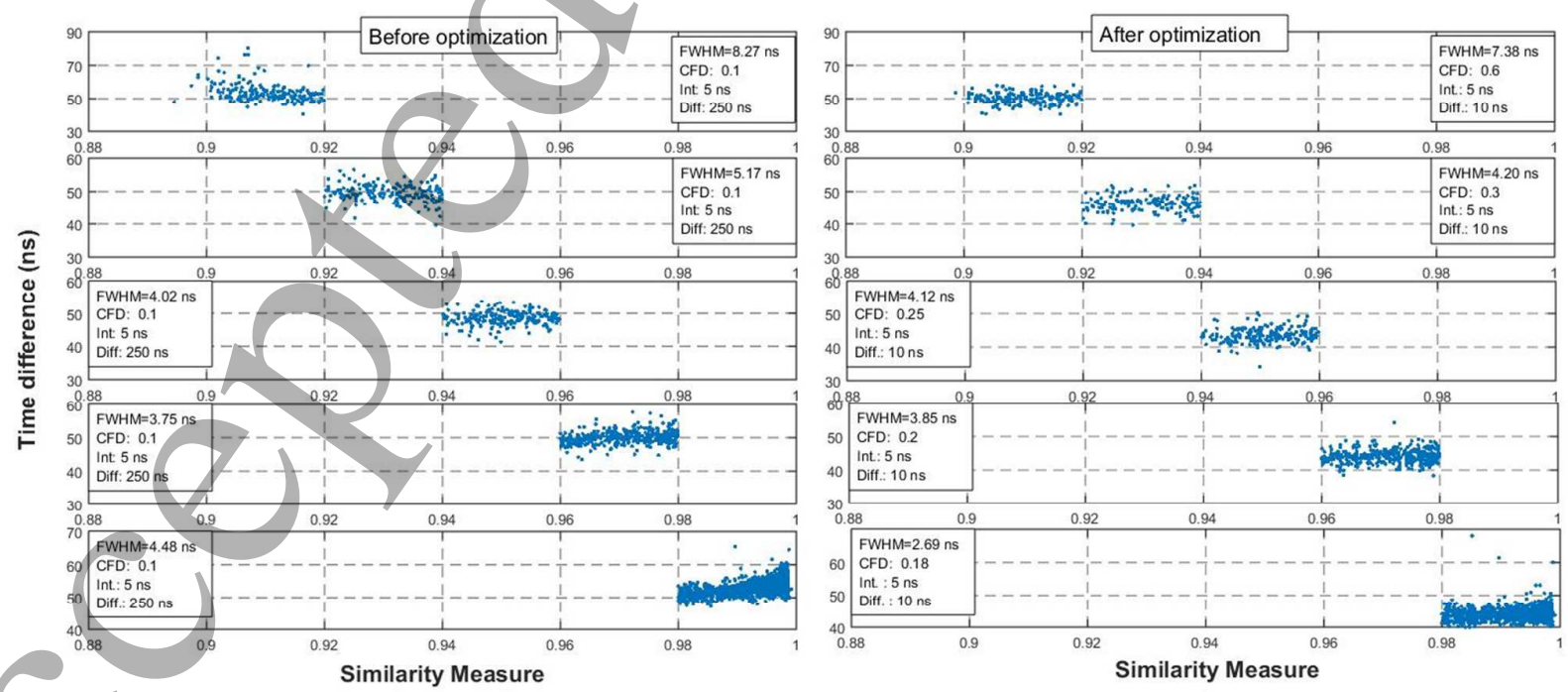

Figure 11. The scatter plots of the time pick-off against the similarity measure for five groups of pulses of similarity measure before and after the optimization of CFD fraction for the minimization of time jitter. The best time resolution for the fastest pulses is obtained as $2.69 \pm 0.04 \mathrm{~ns}$. 


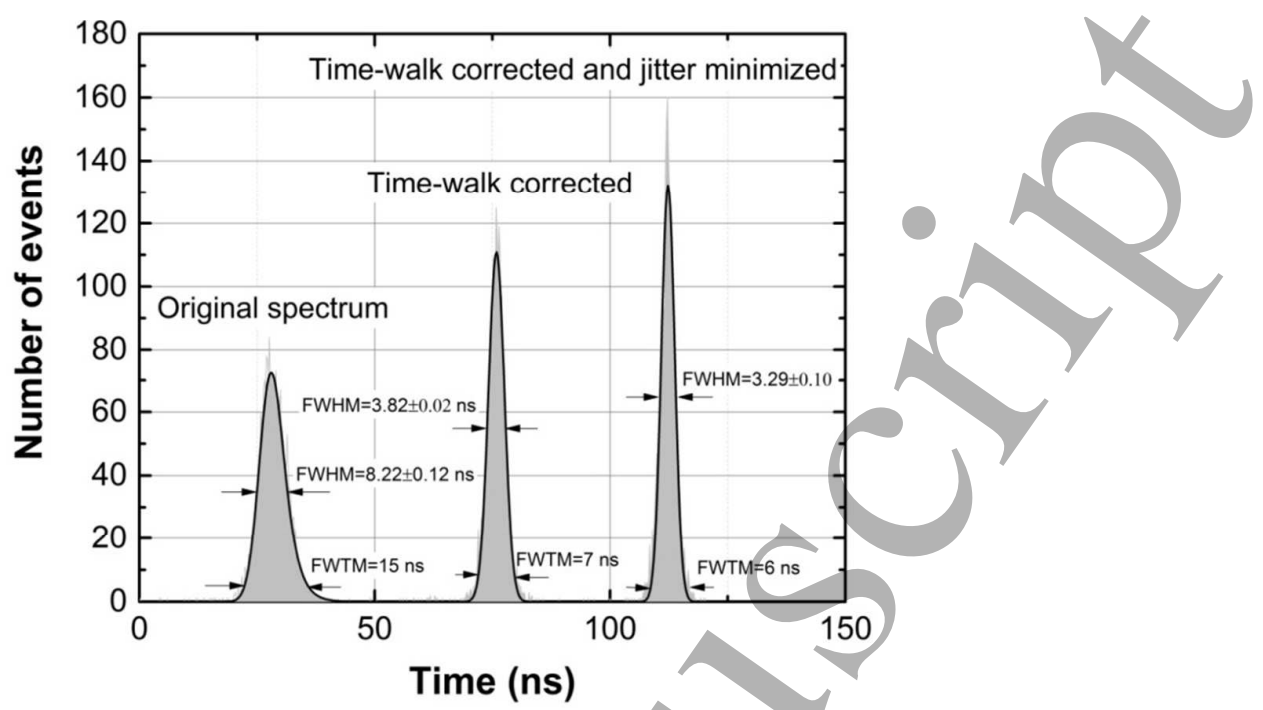

Figure 12. The time-walk corrected and jitter minimized spectrum together with the time-walk corrected and the original time spectra. A time resolution of $3.29 \pm 0.10 \mathrm{~ns}$ (FWHM) is achieved with full detection efficiency.

\subsection{Energy resolution improvement}

In compound semiconductor detectors with planar geometry the energy resolution is mainly limited by the poor charge transport properties of holes. As a result of the small mobility and short lifetime of holes, a fraction of hole charge carriers may be lost during their transit to the cathode. The amount of charge-loss depends on the drift distance of holes, which is determined by the interaction location of $\mathcal{Y}$-rays. Since the shape of pulses reflects the interaction location of $\mathrm{y}$-rays, information on the amount of charge-trapping can be extracted from the shape of pulses as quantified by the cosine similarity measure. Figure 13(A) shows a scatter plot of the amplitude of the pulses against the similarity measure for a ${ }^{137} \mathrm{Cs}$ source. The horizental streak on the top side of the figure represents the full energy deposition events. The amplitude of the full-energy deposition events decreases with decreasing the similarity measure, reflecting a strong correlation between the amount of charge-trapping effect and the similarity measure. It was found that the correlation is best described with a second order polynomial as assessed by the $R$-square value of the fits $\left(R^{2}=0.94\right)$. The fit function was obtained as:

$E(S)=-1744 S^{2}+3665 S-1465$,

where $E$ is the amplitude of the pulses and $S$ is the similarity measure. This relation was used to correct the amplitude of pulses acquired with both ${ }^{22} \mathrm{Na}$ and ${ }^{137} \mathrm{Cs} \gamma$-ray sources. The charge-trapping effect was corrected as $A_{c}(S)=A(S)+(E(1)-E(S))$, where $A_{c}$ is the corrected 


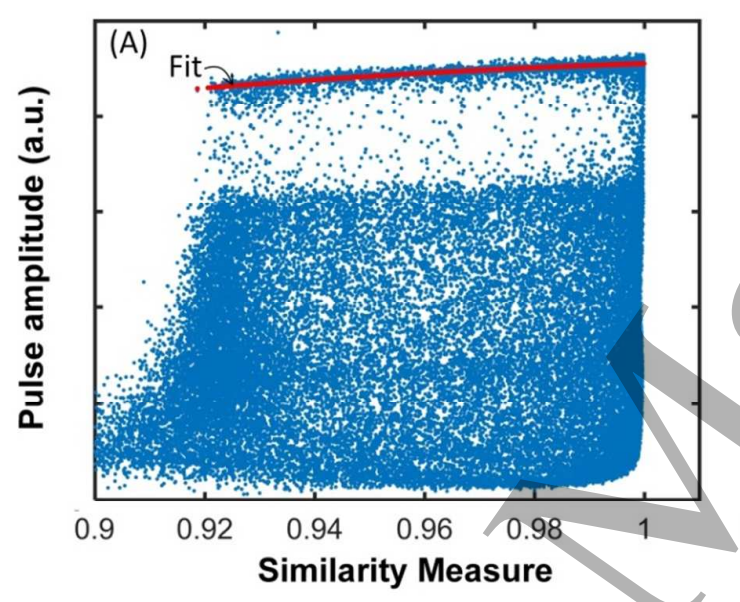

amplitude of the pulse and $A$ is the measured amplitude of the pulse. The corrected scatter plot is shown in figure 13 (B). The corrected energy spectra of ${ }^{137} \mathrm{Cs}$ and ${ }^{22} \mathrm{Na}$ are shown in figure 14. The energy resolution for $662 \mathrm{keV} \gamma$-rays improves from $3.39 \pm 0.07 \%$ to $2.01 \pm 0.02 \%$ (FWHM) and for $511 \mathrm{keV} \gamma$-rays the energy resolution improves from $4.83 \pm 0.66 \%$ to $2.780 \pm 0.002 \%$ (FWHM). It is worth mentioning that the correction of charge-trapping effect also has an effect on the number of recorded coincidence events. Since the charge-trapping effect shifts the energy spectrum to the low energy range, by correcting the charge-trapping effect, the number of events lying in the energy window $300-550 \mathrm{keV}$ increases by $3.61 \%$.

Figure 13. (A) The scatter plot of the outputs of the digital semi-Gaussian filter against the similarity measure. A strong pulse deficit as a function of the similarity measure is apparent, which is represented by a second order polynomial. The $R$-square of the fit is $\mathrm{R}^{2}=0.94$. (B) The scatter plot after the pulse-height correction.

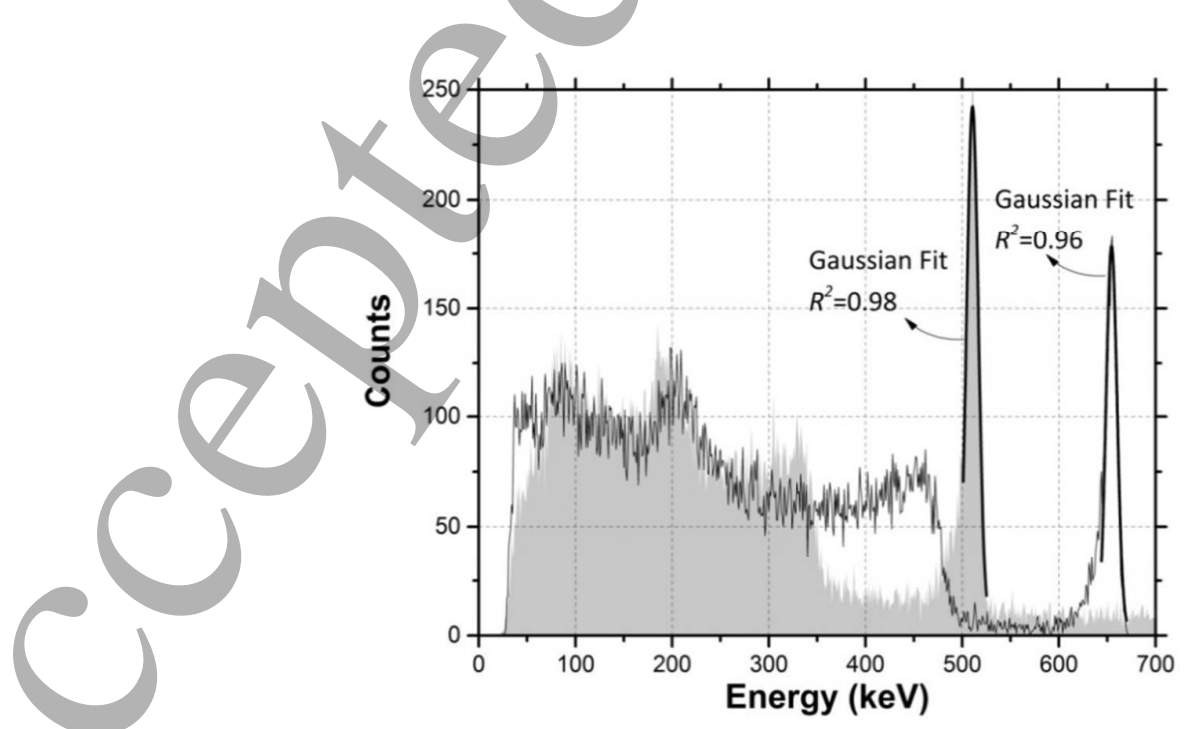

Figure 14. The corrected energy spectra of ${ }^{22} \mathrm{Na}$ and ${ }^{137} \mathrm{Cs}$. The energy resolutions at 511 and $662 \mathrm{keV}$ energy lines as determined by Gaussian fits are, respectively, $2.78 \pm 0.002 \%$ (FWHM) and $2.01 \pm 0.02 \%$ (FWHM). 


\section{Discussion}

\subsection{Prospects for further improvements}

The obtained time resolution of $3.29 \pm 0.10 \mathrm{~ns}(\mathrm{FWHM})$ is in the range of the time resolution of slow scintillator detectors used for PET imaging but obviously further improvement of time resolution will make CdTe detectors more attractive for PET imaging. As it was already discussed this time resolution is practically limited by the level of electronic noise. The electronic noise basically results from three main components: thermal, shot and $1 / f$ noise. The thermal noise results from the input transistor of the preamplifier, the shot noise arises from the detector leakage current and the $1 / f$ noise results from the input transistor and the lossy dielectrics in the front-end. Studies have shown that in Ohmic contact CdTe detectors, operating at room temperature, the shot noise entirely dominates the other sources of electronic noise. We have confirmed this for our detector by measuring the rms value of baseline noise at different bias voltages. At zero bias voltage, the detector's leakage current is non-existent and the noise is due to the thermal and $1 / f$ noise. As the voltage increases the rms value of electronic noise increases due to the leakage current. The rms value of noise at 100 volt increases by a factor of six which shows the leakage current dominates the thermal and $1 / f$ noise. Several studies have shown that leakage current can be significantly reduced by a moderate cooling of the detector. For example, Chirco et al showed that down to $5^{\circ} \mathrm{C}$ the noise continuously reduces by cooling the detector (Chirco et al 1996). Redus et al suggested an optimal temperature of approximately $0{ }^{\circ} \mathrm{C}$ (Redus et al 1996). The measurements by Kastlander and Bargholtz showed that below $0^{\circ} \mathrm{C}$ the leakage current reduces to less than 0.5 $\mathrm{nA}$ as compared to $100 \mathrm{nA}$ at room temperature (Kastlander and Bargholtz 2008). Therefore, further improvement of time resolution would be possible by reducing the leakage current through cooling. Indeed, an improvement of time resolution by a factor of two has been already reported with moderate cooling of $\mathrm{TIBr}$ detectors (Hitomi et al 2011) though the leakage current in $\mathrm{TlBr}$ is considerably lower than that in Ohmic contact CdTe detectors. The results of our study suggest that a sub-nanosecond time resolution could be achieved by reducing the rms value of noise by a factor of 3.5. This is feasible by combining the effects of detector cooling, capacitance matching between the detector and input transistor of the preamplifier and minimization of the stray capacitances between the detector and preamplifier. A sub-nanosecond time resolution makes the CdTe detectors attractive for time-of-flight (TOF) PET applications as well. According to the framework developed for TOF 
PET (Budinger 1983), if the time resolution is improved to $1 \mathrm{~ns}$, then for an object of $40 \mathrm{~cm}$ the improvement in image SNR is about 1.632 as compared to non-TOF PET. Another challenge in the use of CdTe and CdZnTe detectors in PET applications is imposed by the relatively low coincidence detection efficiency of these detectors that has limited these detectors mainly to PET imaging of small animals where high spatial resolution is a priority The intrinsic coincidence detection efficiency depends on several parameters such as the atomic number and density, the length of crystal, packing fraction of detectors and also on the coincidence time window and energy window settings (Peng and Levin 2010b). Our method addresses the low coincidence efficiency of these detectors by improving the timing and energy resolutions that can make these detectors suitable for clinical applications as well. Moreover, the method can be applied to high $\mathrm{Z}$ compound semiconductor $\mathrm{y}$-ray detectors such as $\mathrm{TIBr}$ detectors.

\subsection{Application to other detector geometries}

Our timing analysis and performance improvement has been limited to planar Ohmic CdTe detectors. Our approach is also applicable to strip detectors in which the small pixel effect is negligible, i.e. strip's width is comparable to the detector thickness. However, pixilated and strip detectors with small pixel effect have also attracted some interest for PET applications (Vaska et al 2007, Peng and Levin 2010, Groll et al 2017). In such structures, due to the small pixel effect, the pulse induced on the pixels or strips is entirely due to the motion of electrons at the vicinity of the anode and exhibits larger slopes than that in planar geometry as dictated by the distribution of weighting potential (Barrett et al 1995 and Zumbiehl et al 2001). Moreover, in such detectors, the leakage current is divided between the pixels or strips resulting in lower shot noise. The combined effects of lower noise and larger slope leads to smaller time jitter error as compared to that for planar detectors. However, the timing performance is significantly limited by the fact that the electrons need to travel from the interaction location to the vicinity of the collecting electrode before they can give rise to an output pulse. Due to the random interaction location of $\gamma$-rays in the detector's bulk, the drift time will be variable and this will significantly affect the time resolution. In fact, the timing measurements with pixel detectors have shown time resolutions above 20 ns (Amrami et al 2001, Vaska et al 2007, Groll et al 2017). Since the information on the interaction location is reflected in the shape of induced pulses on the cathode, our pattern 
recognition method could be also used to obtain information on the drift time of electrons, thereby correcting for the measured times of interactions.

\section{Summary}

In this paper, the timing performance of a planar CdTe detector with standard pulse timing method of semiconductor $\mathrm{y}$-ray detectors was studied through simulation and experimental measurements. It was shown that the timing performance is limited by (i) the time jitter mainly results from the leakage current of the detector, (ii) the time-walk error due to the variations in the electron component of the pulses that also produces a tailed time response and (iii) the loss of coincidence events when the parameters of the timing method are adjusted for the best time resolution. A simple cosine similarity measure was employed to quantify the variations in the shape of the pulses, thereby correcting for the time-walk error and minimizing the time jitter through the application of a variable threshold CFD timing method. The time resolution of a 1-mm thick CdTe detector was improved from $8.22 \pm 0.12 \mathrm{~ns}$ (FWHM) to $3.29 \pm 0.10 \mathrm{~ns}$ (FWHM) for an energy range of 300-550 keV while a coincidence measurement efficiency close to $100 \%$ was maintained. The correction also led to a symmetric time spectrum with FWTM of 6 ns from original 15 ns (FWTM). Moreover, the cosine similarity measure was used to correct for the charge-trapping effect and an energy resolution of $2.780 \pm 0.002 \%$ at $511 \mathrm{keV}$ was achieved. The prospect for further improvement of the time resolution and the application of the method to other detector structures were also discussed.

Acknowledgement

I sincerely appreciate the efforts of the reviewers who made many helpful suggestions.

\section{References}

Amrami R, Shani G, Hefetz Y, Pansky A, Wainer N 2001 Timing performance of pixelated CdZnTe detectors Nucl. Instrum. and Meth. A 458 772-781

Baldazzi G, Bollini D, Casali F, Chirco P, Donati A, Dusi W, Landini G, Rossi M and Stephen J B 1993 Timing response of CdTe detectors Nucl. Instrum. and Meth. A 326 319-324 
Barrett H H, Eskin J D, Barber H B, 1995 Charge Transport in Arrays of Semiconductor Gamma-ray Detectors Phys. ReV. Lett. 75 156-159

Budinger T F, 1983 Time-of-flight positron emission tomography: status relative to conventional PET J. Nucl. Med. 24 73-78.

Chirco P, Zanarini M, Caroli E, Donati A, Dusi W, Stephen J B, DiCocco G 1996 Comparative evaluation of the temperature dependence of different noise sources in CdTe detectors Nucl. Instrum. Meth. A 380 127-131

Chase R L 1968 Pulse Timing System for Use with Gamma Rays on Ge(Li) Detectors Rev. Sci. Instrum. 39 1318-1326

Cho Z H, Chase R L 1972 Comparative study of the timing techniques currently employed with Ge detectors Nucl. Instrum. Meth. 98 335-347

Cha S 2007 Comprehensive Survey on Distance/Similarity Measures between Probability Density Functions, International Journal of Mathematical Models and Methods in Applied Sciences Issue 4 Vol. $1300-307$

Crespi F C L, Vandone V, Brambilla S, Camera F, Million B, Riboldi S, Wieland O, 2010 HPGe detectors timing using pulse shape analysis techniques, Nucl. Instrum. Meth. A 620 299-304

Eisen Y, Shor A, Mardor I, 1999 CdTe and CdZnTe gamma-ray detectors for medical and industrial imaging systems Nucl. Instrum. Meth. A 428 158-170

Eriksson L, Townsend O, Eriksson M, Melcher C, Schmand M, Bendriem B, Nutt R 2004 Experience with scintillators for PET: towards the fifth generation of PET scanners Nucl. Instrum. Meth. A 525 242-248 
Groll A, Kim K, Bhatia H, Zhang J C, Wang J H, Shen Z M, Cai L, Dutta J, Li Q, Meng L J 2017 Hybrid Pixel-Waveform (HPWF) Enabled CdTe Detectors for Small Animal Gamma-Ray Imaging Applications, IEEE Trans. Nucl. Sci. Vol.1 3-14

Gu Y, Matteson J L, Skelton R T, Deal A C, Stephan E A, Duttweiler F, Gasaway T M, Levin C S 2011 Study of a high-resolution, 3-D positioning cadmium zinc telluride detector for PET Phys. Med. Biol. 56(6) 1563-1584.

Giboni K, Aprile E, Doke T, Hirasawa M, Yamamoto M 2000 Coincidence timing of Schottky CdTe detectors for tomographic imaging Nucl. Instrum. and Meth. A 450 307-312

Gunnink R, Arlt R 2001 Methods for evaluating and analyzing CdTe and CdZnTe spectra, Nucl. Instrum. Meth. A 458 196-205.

Hecht K 1932 Zum Mechanismus des lichtelektrischen Primärstromes in isolierenden Kristallen Zeitschrift für Physik 77, 235-245

Hitomi K, Tada T, Kim S Y, Wu Y, Tanaka T, Shoji T, Yamazaki H, and Ishii K 2011 Recent Development of TIBr Gamma-Ray Detectors IEEE Trans. Nuc. Sci. 58 1987-1991

Iniewski K (Editor) 2010 Semiconductor Radiation Detection Systems CRC Tailor and Francis Group New York, USA.

Ishii $\mathrm{K}$ et al 2007 First achievement of less than $1 \mathrm{~mm}$ FWHM resolution in practical semiconductor animal PET scanner Nucl. Instrum. Meth. A 576 435-440

Kastlander J, Bargholtz C 2008 Investigation into the properties of CdTe detectors for in-situ measurements Nucl. Instrum. Meth. A 596 409-416

Kikuchi Y, Ishii K, Yamazaki H, Matsuyama S, Yamaguchi T, Yamamoto Y, Sato T, Aoki Y, Aoki K, 2005 Preliminary report on the development of a high resolution PET camera using semiconductor detectors Nucl. Instrum. Meth. B 241 727-731. 
Mitchell G S, Sinha S, Stickel J R, Bowen S L, Cirignano L J, Dokhale P, Kim H, Shah KS and Cherry S R 2008 CdTe strip detector characterization for high resolution small animal PET IEEE Trans. Nucl. Sci. $55870-876$

Morimoto $Y$ et al 2011 Development of a 3D Brain PET Scanner Using CdTe Semiconductor Detectors and Its First Clinical Application IEEE Trans. Nucl. Sci. 58 2181-2189

Meng L J, He Z 2005 Exploring the limiting timing resolution for large volume CZT detectors with waveform analysis Nucl. Instrum. Meth. A 550 435-445

Nakhostin M, Oishi T, Baba M 2008 Time resolution measurement of avalanche counters using digital signal processing technique Radiation Measurements 43 1493-1497

Nakhostin M, Ishii K, Kikuchi Y, Matsuyama S, Yamazaki H, Esmaili-torshabi A 2009 Time resolution improvement of Schottky CdTe PET detectors using digital signal processing Nucl. Instrum. Meth. A 606 681-688

Nakhostin M, Walker P M, Sellin P J, 2010 Time walk correction of CdTe detectors using depth sensing technique Nucl. Instrum. Meth. A 621 506-512

Nakhostin M 2011 Recursive Algorithms for Real-Time Digital CR-(RC) ${ }^{\mathrm{n}}$ Pulse Shaping IEEE Trans. Nucl. Sci. 58 2378-238

Nakhostin M, Podolyak Zs and Regan P H 2014 Digital processing of signals from $\mathrm{LaBr}_{3}: \mathrm{Ce}$ scintillation detectors, Journal of Instrumentation Proceedings of $10^{\text {th }}$ International Conference on Position Sensitive Detectors

Nakhostin M, Esmaili-Torshabi A, 2015 The influence of electron track lengths on the $\mathrm{y}$-ray response of compound semiconductor detectors, Nucl. Instrum. and Meth. A Vol. $797255-$ 259 
Okada Y, Takahashi T, Sato G, Watanabe S, Nakazawa K, Mori K, Makishima K, 2002 CdTe and CdZnTe detectors for timing measurements IEEE Trans. on Nucl. Sci. 49 1986-1992

Origin, 2015 OriginLab, Northampton MA.

Peng H, Levin C S 2010a Design study of a high-resolution breast-dedicated PET system built from cadmium zinc telluride detectors Phy. Med. Biol. 55 2762-2788

Peng H, Levin C S, 2010b Recent Developments in PET Instrumentation, Current Pharmaceutical Biotechnology 11 555-571.

Richter M and Siffert P 1992 High resolution gamma ray spectroscopy with CdTe detector systems Nucl. Instrum. and Meth. A 322 529-537

Redus R, Squillante M, Lund J 1996 Electronics for high resolution spectroscopy with compound semiconductors Nucl. Instrum. Meth. A 380 312-317

Redus R H, Pantazis J A, Pantazis T J, Huber A C, Cross B J, 2009 Characterization of CdTe Detectors for Quantitative X-ray Spectroscopy, IEEE Trans Nuc. Sci. 56 2524-2532.

Seino T, Ishitsu T, Ueno Y, Kobashi K, 2011 Biparametric correction methods using two shapers for In/CdTe/Pt radiation detector Nucl. Instrum. Meth. A 629 170-174

Takenaka Y, Uritani A, Inoue K, Sakai H and Mori C 1998 Novel Pulse-shape Analysis Method by Using Similarity Appl. Radiat. Isot. Vol. 49 1219-1223.

Ueno Y, Morimoto Y, Tsuchiya K, Yanagita N, Kojima S, Ishitsu T, Kitaguchi H, Kubo N, Zhao S, Tamaki N, Amemiya K 2009 Basic Performance Test of a Prototype PET Scanner Using CdTe Semiconductor Detectors IEEE Trans. Nuc. Sci. 56 24-28

Vaska P, Bolotnikov A, Carini G, Camarda G, Pratte J F, Dilmanian F A, Park S J and James R B 2005 Studies of CZT for PET applications IEEE Nucl. Sci. Symp. Conf. Rec. 5 2799-802 
Vaska P, Dragone A, Lee W, Kim D. -H, Pratte J. -F, Cui Y. -G, Fried J, Krishnamoorthy S, Bolotnikov A, Park S. -J, O'Connor P, Dilmanian F A, James R B, 2007 A prototype CZT-based PET scanner for high resolution mouse brain imaging IEEE Nucl. Sci. Conference 3816-3819

Wilmshurst T H 1985 Signal Recovery from Noise in Electronic Instrumentation (Brístol: Hilger).

Zhang F, Zhong H, Xu D and Meng L J 2005 Feasibility study of using two 3D position sensitive CZT detectors for small animal PET IEEE NUcl. Sci. Symp. Conf. Rec. 3 1582-1585

Zumbiehl A, Hage-Ali M, Fougeres P, Koebel J M, Regal R, Rit C, Ayoub M, Siffert P 2001 Modelling and 3D optimisation of CdTe pixels detector array geometry -Extension to small pixels Nucl. Instrum. and Meth. A 469 227-239 